formacion; dew Langerhansschen „Inseln", erzeugt und an die Blutbahn abgregeben wird. Banting in Toronto ist es gelungen, die wirksame Substanz, wm deren Auifindung sich viele vergeblich bemlidht hatten, das ,Insulin", xein zu gewinnen. In einem formpollendeten Vortrage, mit dem der Longrel3 eröffuet wurde, berichtete der Leiter des Instituts von Toronto, Mac Leod, zusammentassend über die Ermobnisse, die er un seine Mitarbeiter bisher mit dieser wunderbaren Substanz erzielt haben, von der wir anscheinend nicht blof ungeahnte Autscblüsse über den Zellchemismus, sondern auch ein wichtiges Mittel für die Behazdlung der Zuckerkrankheit erhoffen dürfen. Gegenüber diesem Neulani wissenschafticher Forschung traten die Fortschritte in der Beackerung schon lange bestellter Felder mehr in den Hintergrund. Von den Mitteilungen a'eutscher Forsoler seien besonders hervorgehoben die interExsanten Ausfuhrungen Rubners uber den Zusammenhang von Wachstum und Wassergehadt des Organismus und der experimentelle Nachweis Warburgs, daB der EinfluB der verschiedenen Lichtstrahlen aux die pflang. lieho Assimilation der Quantentheorie folgt.

Die Demonstrationen, sonst mit dev" wertwoluste Teil eines Eongresseis, blielben trotz ibrer gleiehfalla beträchtlichen Zahl an Bedeutung zurück, and da die deutsche Mechanikerkunst nicht rertreten war, suchts nıan veroublich nach größeren technischen Errungenschaften. Vier Filme erregten großns Interesse: Ein russischer Film schilderte anschaulich die Lebensg:wohnheiten eines grobhirnlasen Hundes, Krogh (Kopenlagen) zeigte seine mit dem Nobelpreis gekrönte Beobachtung der wechselnden Blutdurchströmung eines Organs infolge Anderumg der Weite der Kapillarein, Frank (München) eine neue ingeniöse Methodik, dre tine Analyse der Herzbewegungen ron bisher unar. reichter Präzision gestattet. und ein Pariser Film lemonstrierte in zun Sehlub etwas vaudevillemäBjer Aufmachung eine Verjungung beim Menschen dareh Iransplantation von Chimpansenhodea.

Außer der Eröffnungssitzung vereinigten noch zW gemeinsame Sitzungen die KongreBteilnehmer: Richets meisterhafte Rhetorik. war sicher ein hoher akustischeir GenuB; daB aber die Telepathi'e eine experimentelle Wissenschaft sei, die in den Bahmen $C l$. Bernards uvd O. Luduigs wandle, diese Uberzengung wird wohl ails seinen Ausfihrungen auch der nicht davongetragen iwaben, der diesen Enscheiwumgen mit gröbtem Interesic and völliger Unvoreingenommenheit gegenubersteht una inre vorurteilslose Untersuchung fur eine dringende Fordlerung halt. Die schlidisitzung, in der, mit großem Beifall begrüist, der Altmeister der russisehen Physiologie, Pawlow, durch den Mund seines Sohnes über rie reuen Ergebuisse seiner Arbeiten berichten ließ, konnte uns Deutschen die tröstliche Zuversicht geben, daß d:e wissensehaftliche Forschung auch die ärosten Wirrea siegreich" zu überdawern vermag. Hans Winterstein.

\title{
Physiologische Mitteilungen.1)
}

Eine Vorlesung iber die Physiologie des Insulin. (II. A. Dale, lancet Bd. 204, Nr. 20, S. 989-993, 1923.) Nach einer historischen Einleitung wurden besprochen 1. die Langerhansschen Inseln alis Quelle einer inneren Sekretion. Die Frage, ob die Langerhannsschen Inseln beim menschlichen Diabetes veränzert sind, kounte nicht gelöst werden, weil das Untersuchungismaterial ganz frisch sein muß, dagegen gelang es Allen nachzuweisen (im Tierversuch mit den Färbemethoden von Bensley und Lanej, daß die $\beta$-Zellen der Lamgerhansschen Inseln im Diabetes ihre Granula verheren und walknolisiert werden. Trotzdem gelang es vielen Untersuchern nicht, das wirksame Prinzip $z u$ isolieren. 2. Die Entdieckung des Insulins durch Baiting und. Best, welche die sezernierenden Zellen des Pankreas durch Gangunterbinłlung zerstörten und aus dem Drǚsenrest durch Salzlösung das wirksame Prinzip extrahierten und seine Wirbsamkeit auf den Blutzucker des pankreasdiabetischen Hundes nachwiesen. Dann gelang auch die Darstellung aus Pankreas von geschlachtecen Tieren, durch fraktionierte Alkoho? fülung. 3. Die chemische Natur des Insulins, Es ist nicht chemisch rein dargestellt, aber bereits in sehr stark wirksamen Präparaten. Schon 0,25 mgi Substanz können am Kaninehen die charakteristischen Krampfe hervorrufen. Da esi also eime höchst adsorbierte Substanz ist, so ist die wirklich wirkende Menge wohl noch viel kleiner. Dudley fanin daß ein durch Pikrinsäure erzeugter Niederschlag alles Tnsulin aus seinen Lösungen mitreißt. Es ist wahrscheinlich ein höchst komplexes Eimeißderivat. Es wird durch Pepsin und Trypsin zerstört. Daher ist seine Anwendung bei der Therapte nur durch Tijektion möglich, nicht durch $\mathrm{Zu}$ fubr per os. In saurer Lösung kann es $1 / 2$ Stunde auf $100^{\circ}$ erhöht werden, whe zerstört zu werden. In aldallsober Lösung ist es nicht haltbar. Die Erkennung seiner Struktur ist sehr schwierig, zunächst kann nur auf

1) Aus den Bierichten ïber idlie gesamte Physiologie und exrerimentelle Pathologie. reine Darstellung stabiler Präpwate gehofft werden. 4. Sein Vorkommen in der Natur. Beit dea Wirbeltieren Irommt es nur in dien Langerhansschen Insein des Pankrears vor, doch haben Winkler und Smith and Collip es auch in Substanzen mit insulinähnlicher Wirkung in Hefe, rasch wachienden Pflanzen, Muscheln nachgerwiesen. 5. Seine Fuligkeit, die innere Sekretion des Pankreas zu ersetzen. Beim pankreasdiabetischen Tier bringt es die Erscheinungen des Diabetes rum BewuBtsein, beim normalen Tier senkt os den Blutzuaker, aber nicht Jurch unmitte har vermehrte Zuekerverbrennung. 6. Der Zusammenhang der Insulinwirking mit der Zuckerverbrennung. Nach Insulingabe steigt zuä̈chst der R. Q., aber aur durch vermehrte $\mathrm{CO}_{2}$-Abgabe, wäbrend der Saurerstoffyerbrauch unverändert bieibt. Sinkt also ver Blutzucker, sor sinkt auch der Gaswlechsel tin $1 / 2$ Stunde um $50 \%$ ) und die Körpertemperatur. Wenn der Zucker trotidem verschwindet, muB er in andere Storffe umgewandelt sein. Er wird also nioht zu GTykagen polymerisiert. Auch eine Umwandlung in Fet konnte nicht nackgewiesen werden. Der Zucker venschwindet, aber wir wissen nichb, wie. 7. Der gegenwärtige Stand unserer Kenntnisse. Am meisten mit dem gerenwartigen Stande scheint Date folgende Antahme zu liegen: Insulininjektion bezweckt beim normalen Tier zunächst allein Steigerung der Zuckerverbremung in solchem $\mathrm{MaBe}$, daß Aer Organitsmus an Fohlelydrat so verarmt, daß der Blutzucker sinkt. Dann tritt Sinken des Gaswechsels ein, und statt Zucker wird Eiweis und Fett verbrannt. Die Möghichkeit, daß $\alpha-\beta$-Glukase in $\gamma$-Glukase umgewandelt wird (Winter und smith, Embden-Laquer), scheint Dale weniger in der Lage zu sein, das Verschwinden des Zuckers aus dem Blute zu enklären.

Der Einfluß des Ernährungszustandes des Tieres auf die Insulinhypoglykämie. (N. A. Mc Cornick, $J$. J. R. Hacleod, E. C. Noble and K. O'Brien, Journ. of physiol. Bd. 5\%, Nr. 3/4, S. 234-252, 1923.) Das. 